\title{
Remote Hardware-in-the-Loop Approach for Microgrid Controller Evaluation
}

Kumaraguru Prabakar, Amir Valibeygi, Sai Akhil R. Konakalla, Brian Miller, Raymond A. de Callafon, Annabelle Pratt, Martha Symko-Davies, and Thomas Bialek

Clemson University Power Systems Conference Clemson, South Carolina March 10-13, 2020 


\section{Contents}

1 Energy systems integration facility

2 Remote controller hardware-in-the-loop setup

3 Remote setpoint experiments

4 Remote microgrid controller experiments

5 Summary

6 Future work 


\section{Energy Systems Integration Facility (ESIF)}

The ESIF is a national User Facility located in Golden, Colorado on the campus of the National Renewable Energy Laboratory (NREL).

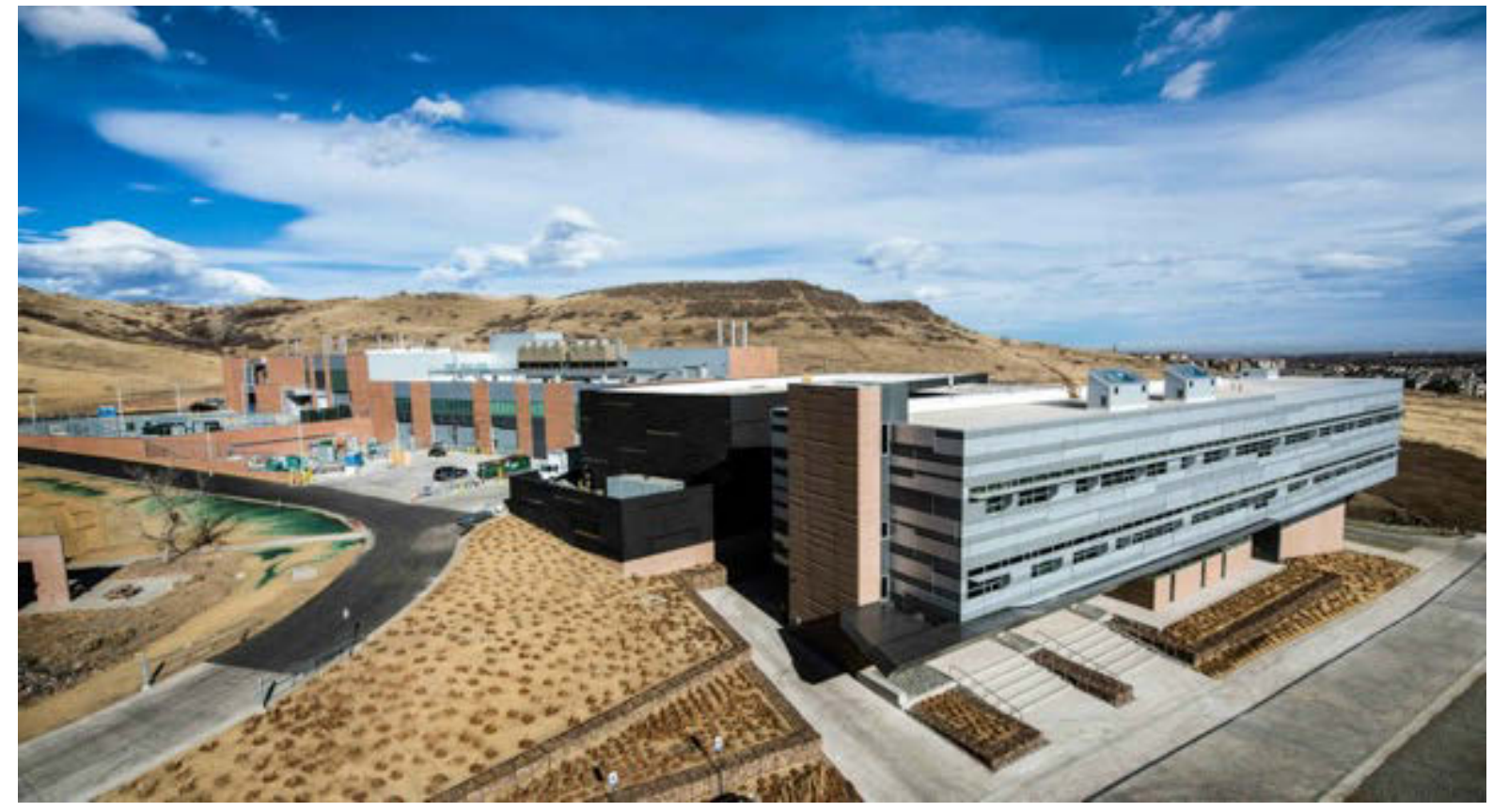

http://www.nrel.gov/esif 


\section{Controller and Power Hardware In The Loop (CHIL/PHIL)}

NREL's megawatt-scale controller and power hardware-in-the-loop (CHIL/PHIL) capability allows researchers and manufacturers to test energy technologies at full power in real-time grid simulations to safely evaluate performance and reliability

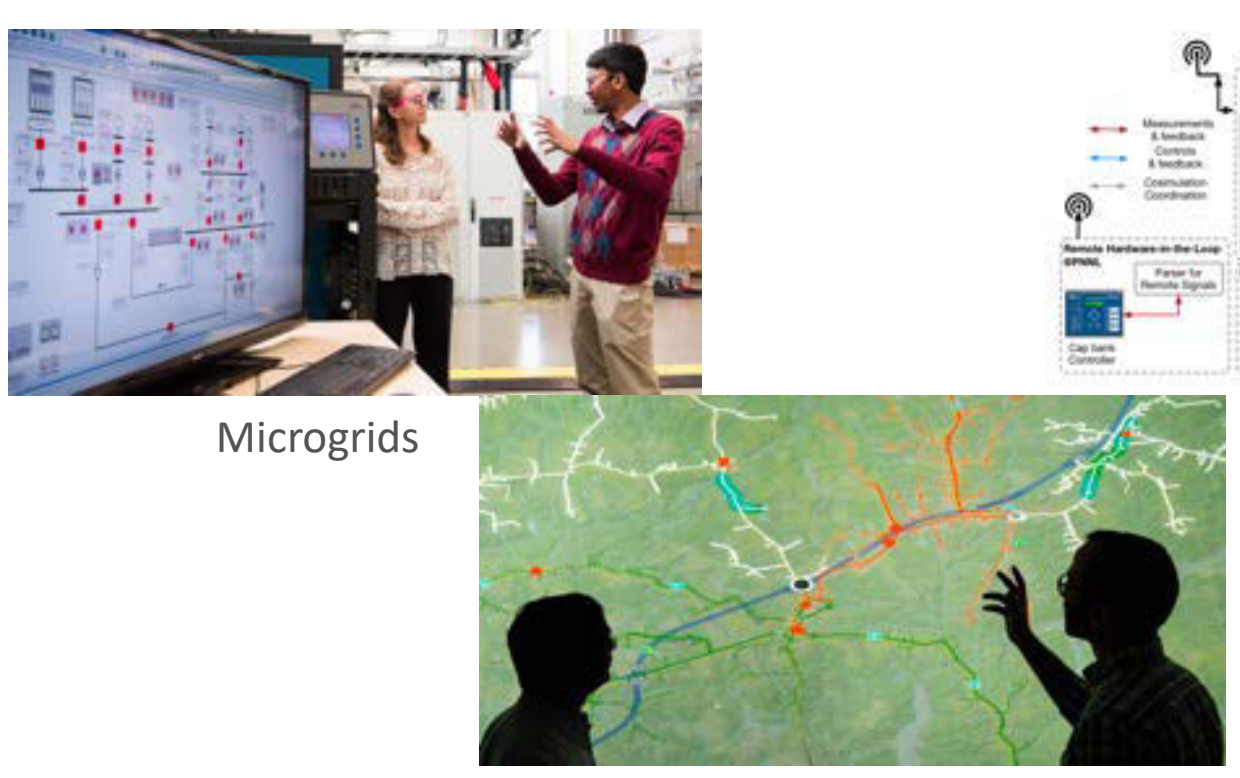

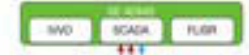

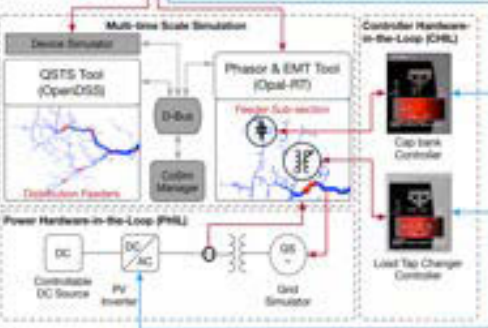

Cosimulation

Power System Studies 


\section{Team information}

- NREL

- Kumaraguru Prabakar

- Brian Miller

- Annabelle Pratt

- Martha Symko-Davies

- San Diego Gas \& Electric Company

- Thomas Bialek
- University of California, San Diego

- Amir Valibeygi, Sai Akhil R. Konakalla Courtney Pailing

- Raymond A. de Callafon 
Hardware-in-the-loop

setup 


\section{Remote controller hardware-in-the-loop setup}

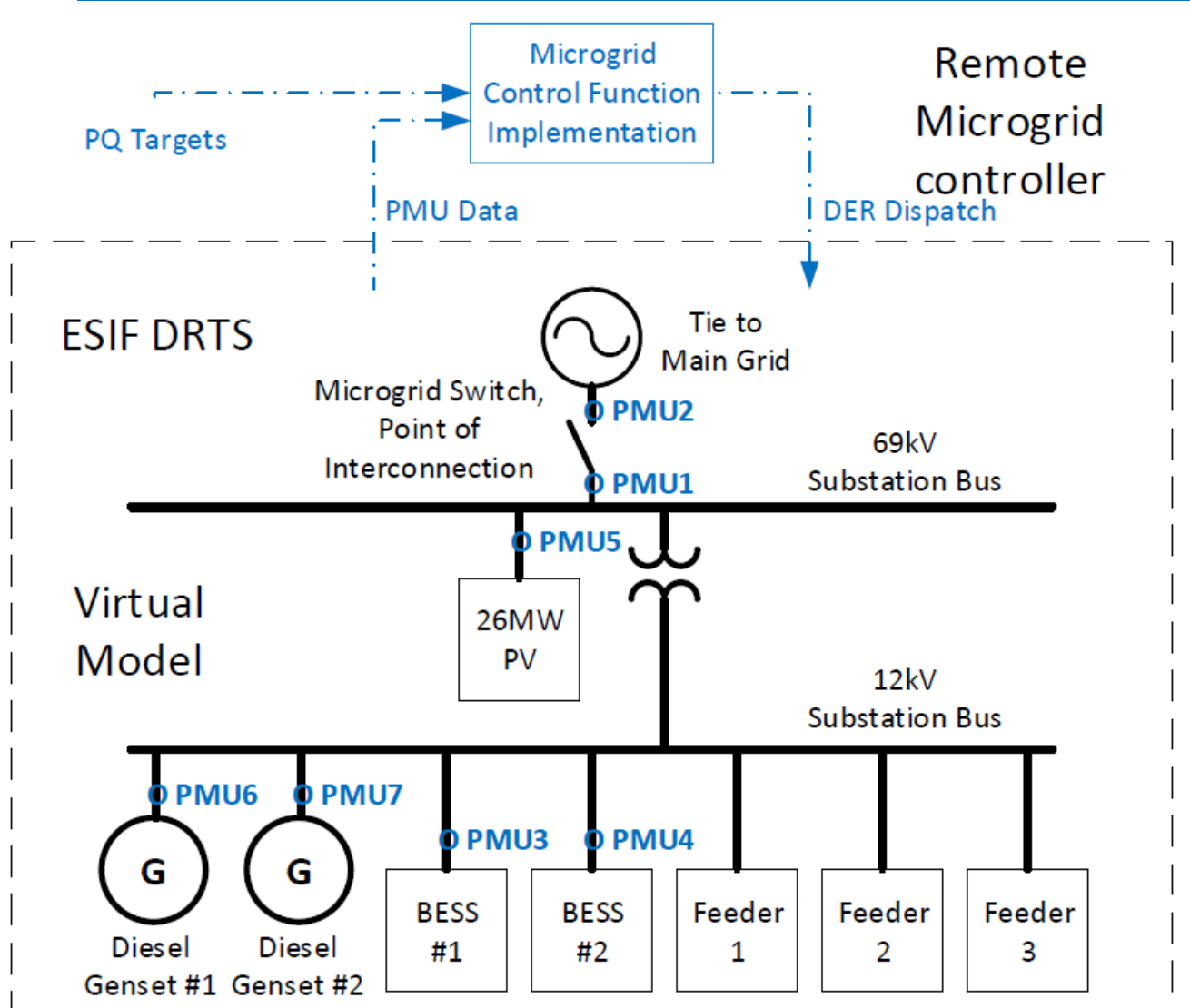

- Five assets

- Two BESS

- Two diesel generators

- One 26 MW PV plant

- One POI circuit breaker

- Seven PMUs 


\section{Remote controller hardware-in-the-loop setup}

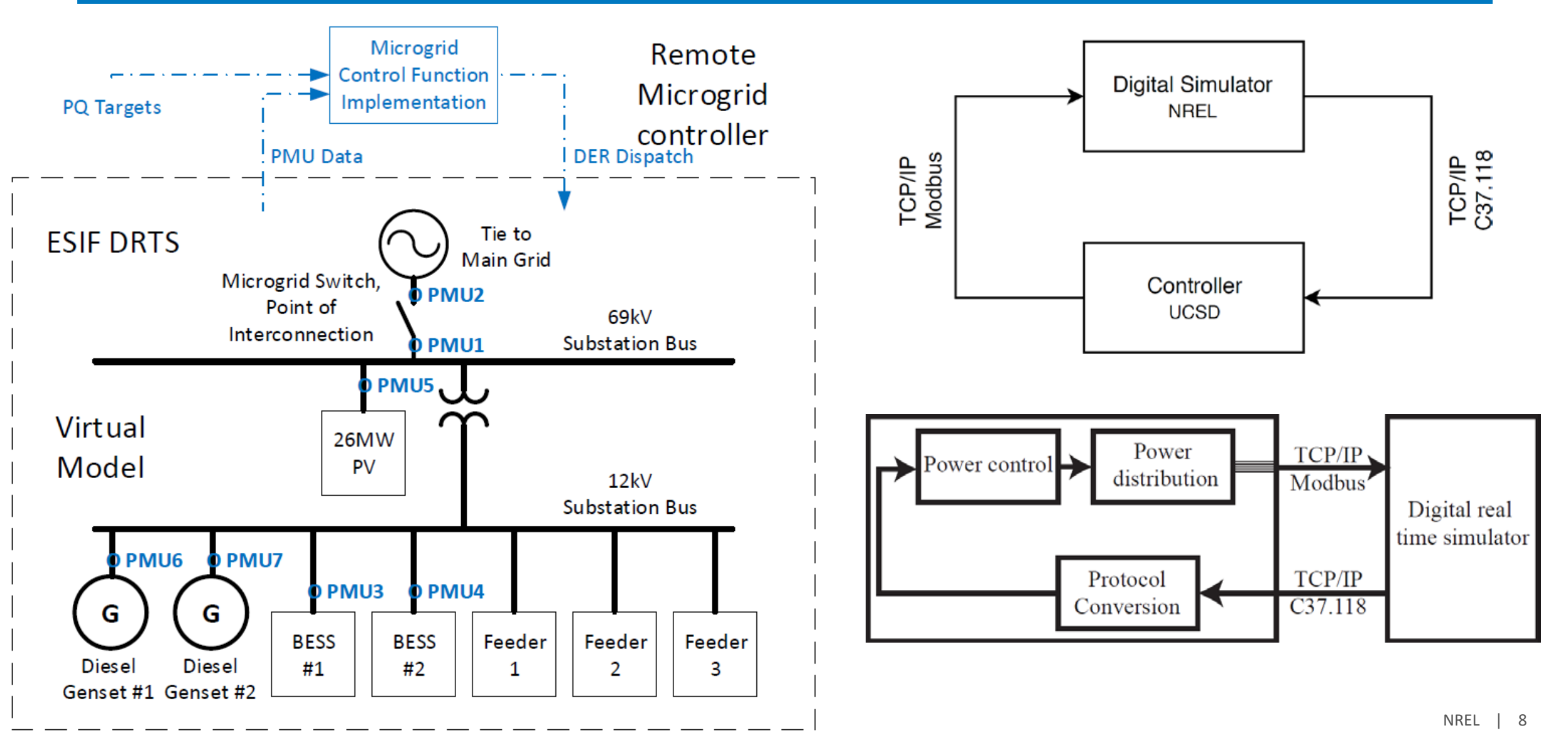




\section{Remote setpoint experiments}
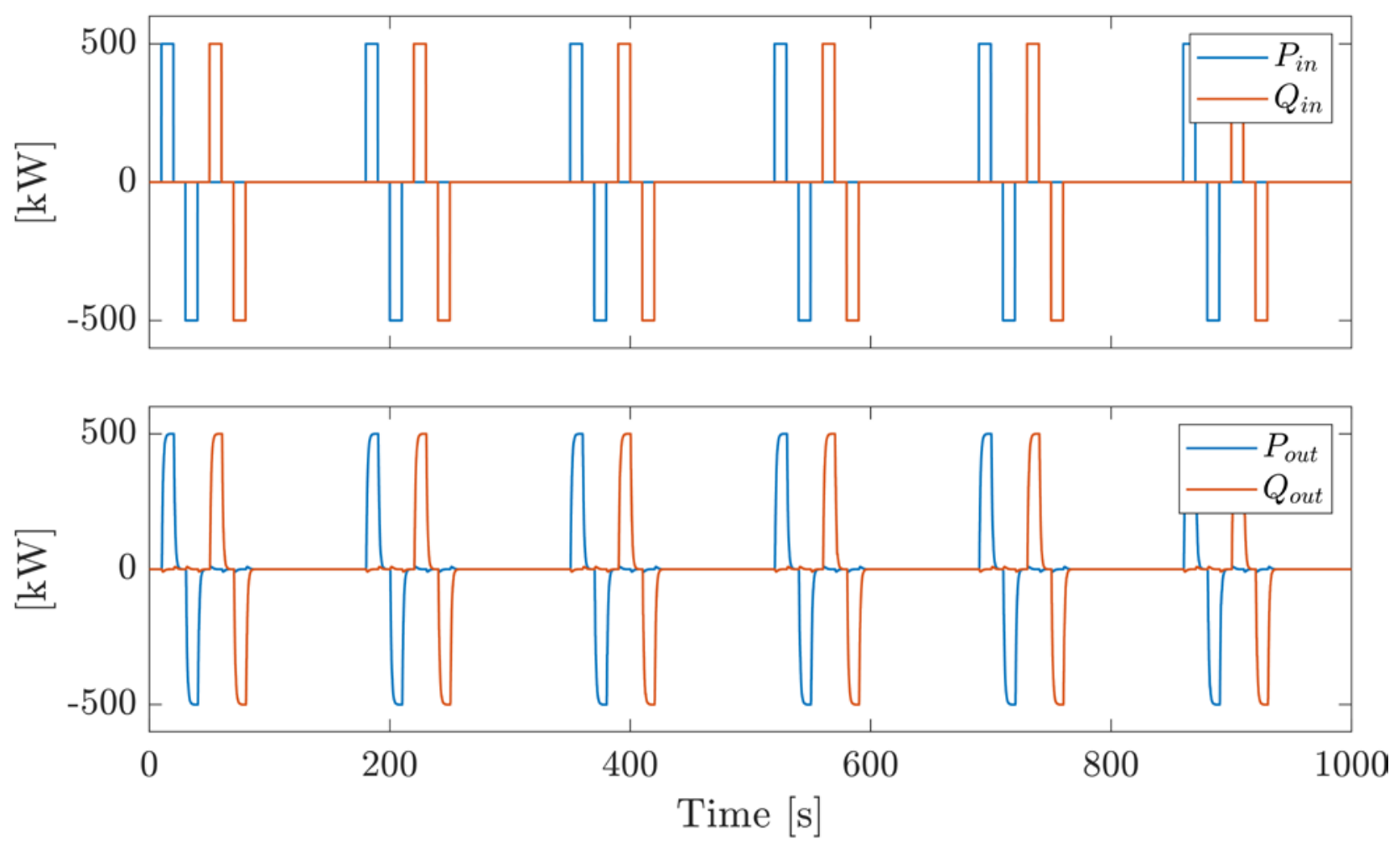


\section{Remote setpoint experiments}
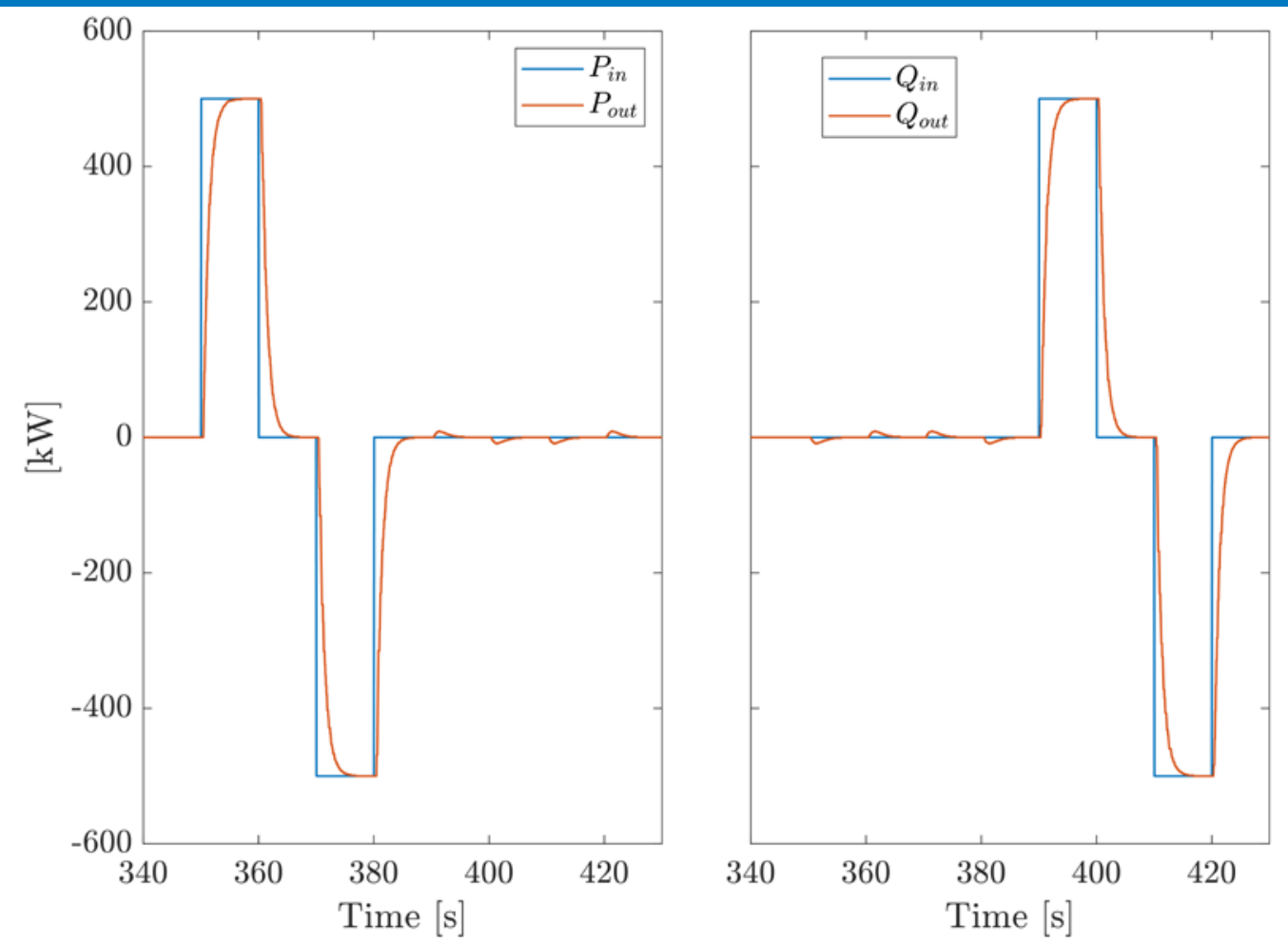


\section{Remote setpoint experiments}
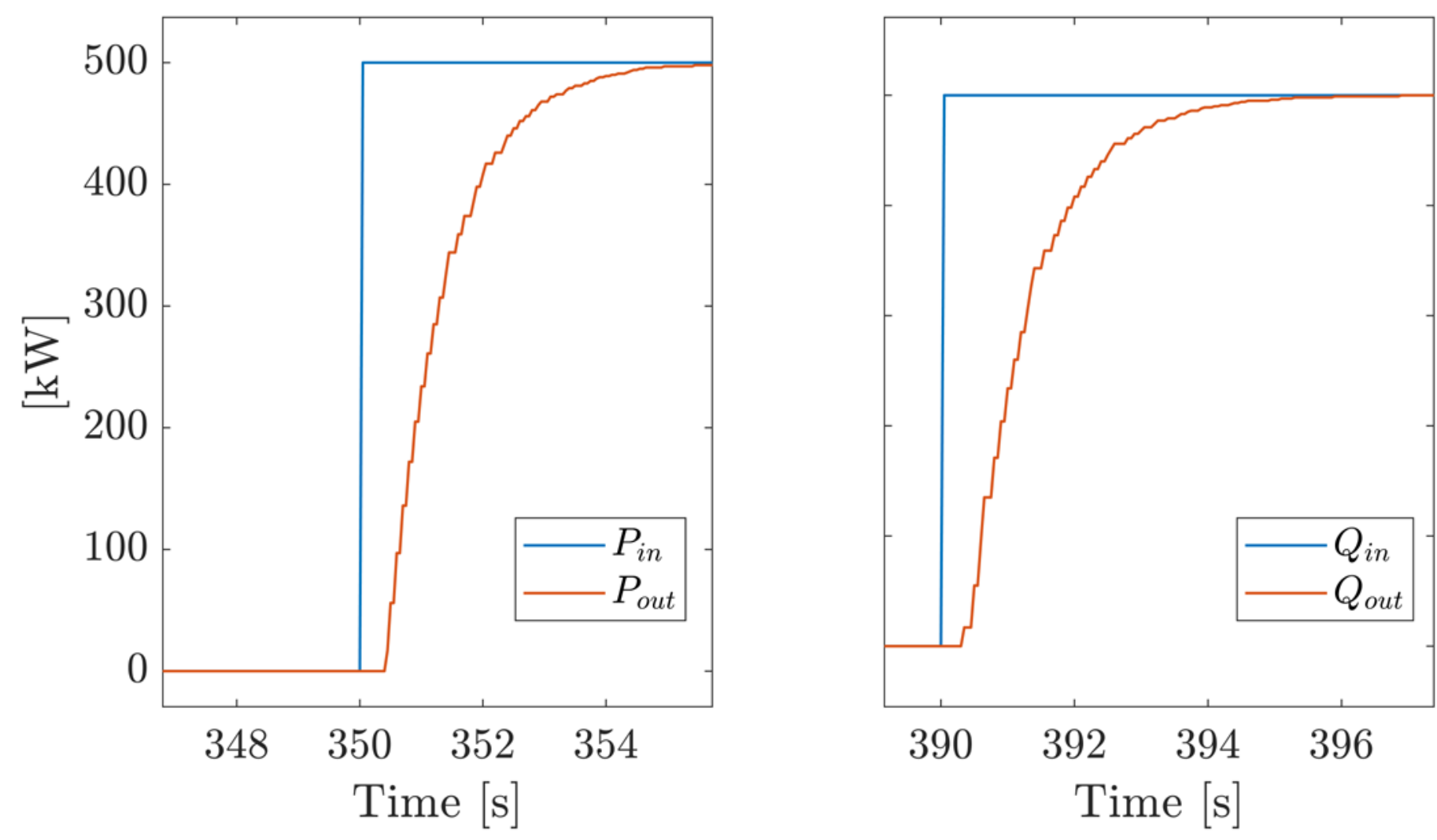


\section{Remote setpoint experiments}

$$
\begin{aligned}
& P_{\text {out }} \\
& =z^{-8}\left[\frac{0.05 z^{-1}-0.047 z^{-2}}{1-1.9 z^{-1}+0.9 z^{-2}} P_{\text {in }}\right. \\
& \left.+\frac{0.002 z^{-1}-0.002 z^{-2}}{1-1.9 z^{-1}+0.9 z^{-2}} Q_{i n}\right]
\end{aligned}
$$

Simulated Response Comparison

$$
\begin{aligned}
& Q_{\text {out }} \\
& =z^{-8}\left[\frac{-0.002 z^{-1}+0.002 z^{-2}}{1-1.9 z^{-1}+0.9 z^{-2}} P_{\text {in }}\right. \\
& \left.+\frac{0.05 z^{-1}-0.047 z^{-2}}{1-1.9 z^{-1}+0.9 z^{-2}} Q_{\text {in }}\right]
\end{aligned}
$$
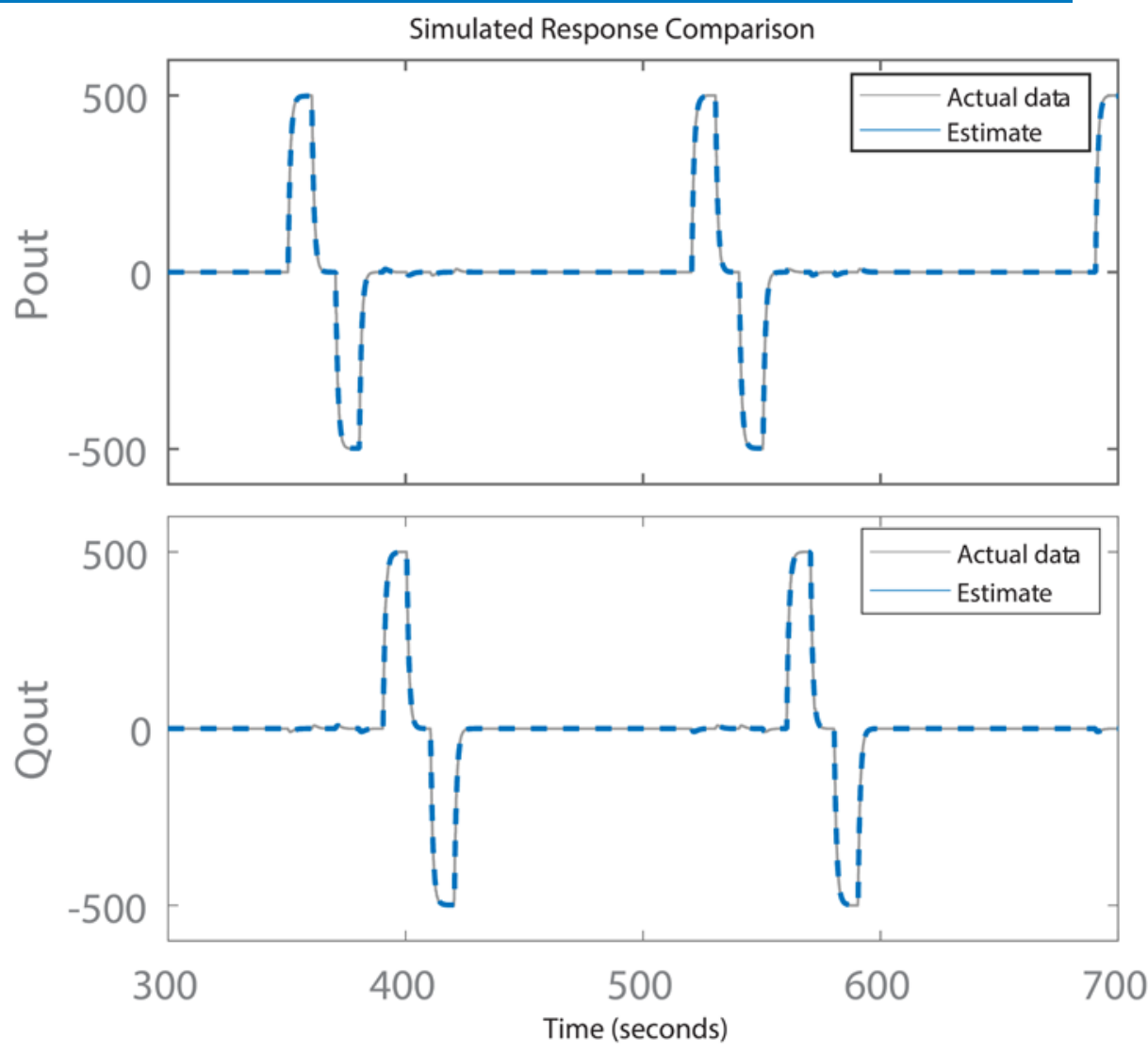
Microgrid controller
experiments

Microgrid co
experiments 


\section{Remote microgrid controller experiments}
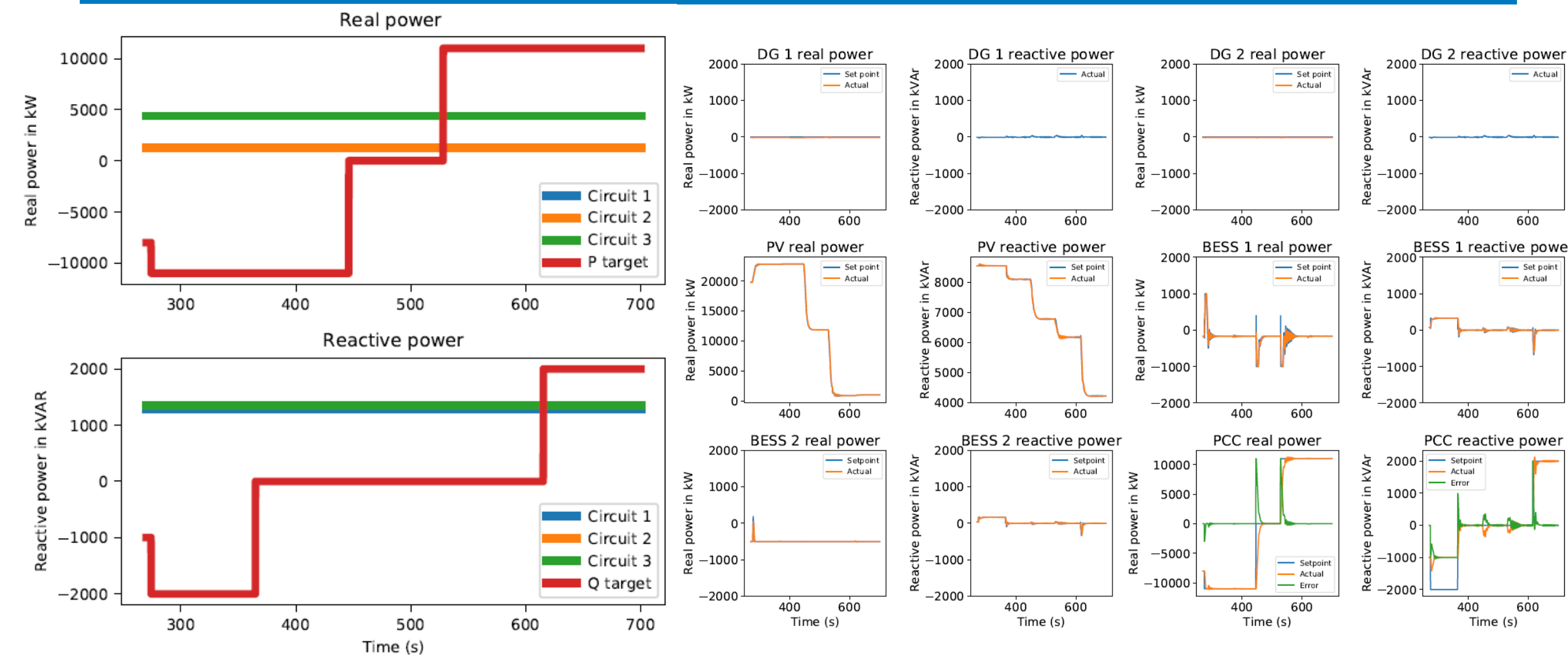


\section{Remote microgrid controller experiments}
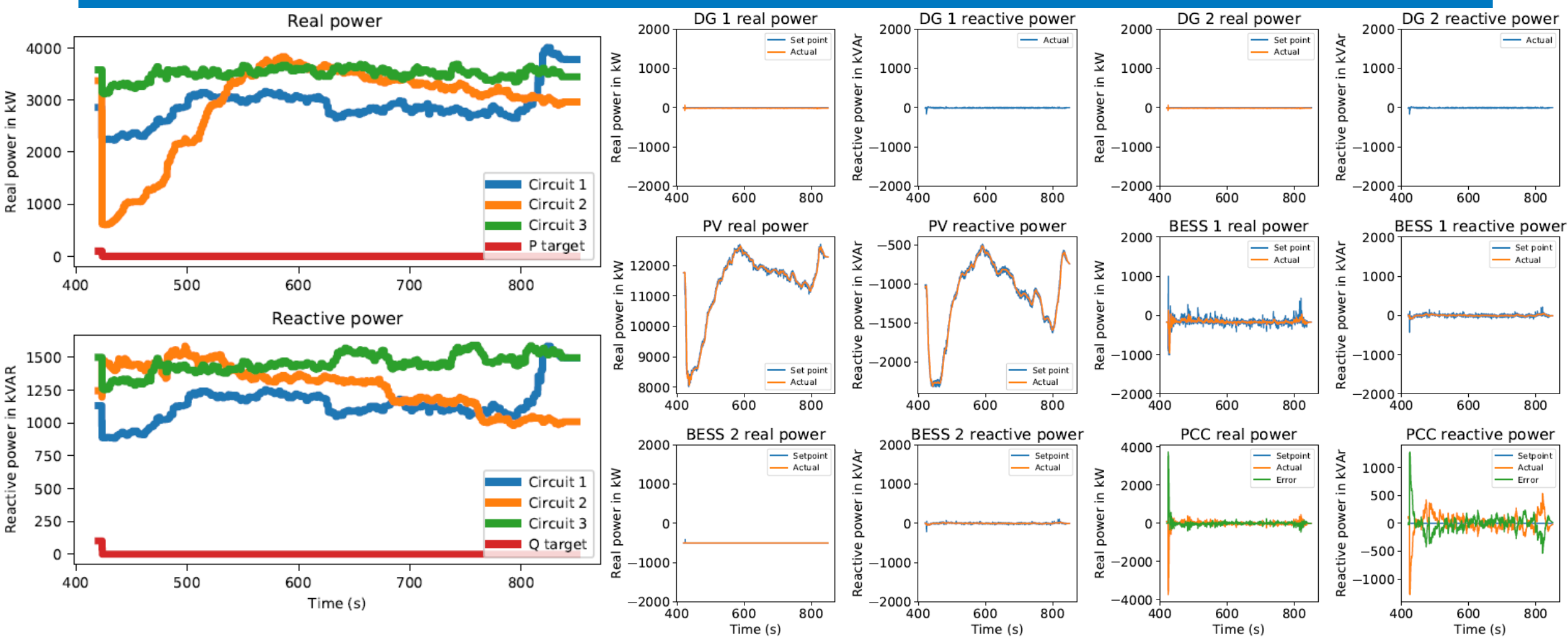


\section{Summary}

- A remote HIL setup was implemented to evaluate a microgrid control function developed by the SyGMA laboratory at UCSD.

- The remote HIL setup consists of a DRTS running a simulation of the microgrid at NREL in Colorado, an implementation of the microgrid control function at UCSD in California, and an internet-based connection between these two location.

- The novelty of the approach is the use of power system communications protocols (C37.118) to exchange information between two different locations.

- By characterizing the effects of networked communications on the closed-loop feedback controller, a control system is designed that sends DER power commands to each simulated DER over the Internet and successfully achieves the objective of following the power set points.

- The remote controller-simulator setup is tested with three test cases, demonstrating successful power control at the POI of the microgrid. 
- 24 hour remote controller hardware-in-the-loop

- Remote power hardware-inthe-loop

- Islanded mode of operation

- PQ following mode to VF master mode (DER operation) 


\title{
www.nrel.gov
}

\author{
NREL/PR-5D00-76279
}

This work was authored in part by the National Renewable Energy Laboratory, operated by Alliance for Sustainable Energy, LLC, for the U.S. Department of Energy (DOE) under Contract No. DE-AC36-08G028308. Funding provided by the California Energy Commission. The views expressed in the article do not necessarily represent the views of the DOE or the U.S. Government. The U.S. Government retains and the publisher, by accepting the article for publication, acknowledges that the U.S. Government retains a nonexclusive, paid-up, irrevocable, worldwide license to publish or reproduce the published form of this work, or allow others to do so, for U.S. Government purposes.

\section{ENREL}

Transforming ENERGY 